\title{
Short-term and long-term results of cardiac surgery in elderly and very elderly patients
}

\author{
Giuseppe Speziale, MD, ${ }^{\mathrm{a}}$ Giuseppe Nasso, MD, ${ }^{\mathrm{a}}$ Maria Cristina Barattoni, ${ }^{\mathrm{b}}$ Giampiero Esposito, MD, ${ }^{\mathrm{d}}$ \\ Georges Popoff, MD, ${ }^{\mathrm{c}}$ Vincenzo Argano, MD, ${ }^{\mathrm{e}}$ Ernesto Greco, MD, ${ }^{\mathrm{h}}$ Marcio Scorcin, MD, \\ Claudio Zussa, MD, ${ }^{\mathrm{b}}$ Donald Cristell, MD, ${ }^{\mathrm{f}}$ Roberto Coppola, MD, ${ }^{\mathrm{g}}$ Sergio Chierchia, MD, ${ }^{\mathrm{g}}$ \\ Alfredo Marchese, ${ }^{\mathrm{a}}$ Pasquale Caldarola, MD, ${ }^{\mathrm{i}}$ Khalil Fattouch, MD, ${ }^{\mathrm{j}}$ and Luigi Tavazzi, $\mathrm{MD}^{\mathrm{b}}$
}

Objective: Cardiac operations in elderly patients are increasingly frequent and imply major clinical, ethical, and economic issues. Operative and 5-year results of cardiac operations in patients aged 79 years or more are known in limited series, and a debate is ongoing on the appropriateness of selection of patients for surgery.

Methods: We retrospectively reviewed our experience in 6802 patients aged 79 years or more who had received
a cardiac operation. Surgical candidates were selected according to functional status, crude operative risk, and social context and were managed according to a multimodality protocol.

Results: Mean age was 82 years and surgery was nonelective in 1613 cases (23.5\%, 31 salvage). Procedures consisted of valve replacement (aortic, 2817; mitral, 532; and tricuspid, 2 cases), valve repair (aortic, 66; mitral, 532; and tricuspid, 232 cases), coronary bypass grafting (12,034 coronary vessels bypassed), and replacement of the thoracic aorta (ascending, 315; arch, 28 cases). Overall operative mortality was 3.4\%. Nonelective presentation, need for aortic counterpulsation, cardiopulmonary bypass time, blood transfusion, depressed systolic function, and chronic lung disease predicted operative mortality. Five-year cumulative mortality was $7.5 \%$. Poor systolic function, previous myocardial infarction, and combined coronary/mitral surgery predicted late mortality. The operative risk of nonagenarians operated on electively did not differ from that of risk-matched octogenarians.

Conclusions: Cardiac surgery in elderly and very elderly patients can be performed with acceptable mortality provided that accurate selection and a multifactorial risk evaluation are adopted. Whenever possible, nonelective operations should be avoided and earlier surgery should be encouraged. Five-year survival and functional recovery are good. (J Thorac Cardiovasc Surg 2011;141:725-31)

\section{Supplemental material is available online.}

Driven by the increasing age of both the general population and the population of patients affected by heart disease ${ }^{1,2}$ and by the progress of intraoperative and postoperative care, cardiac operations in the octogenarians are nowadays a clinical reality. Most of the available data are derived from aortic valve replacement interventions ${ }^{3-5}$; aortic valve disease and coronary disease are the most frequent indications for cardiac surgery in elderly patients. There is

\footnotetext{
From the Cardiac Surgery Departments of GVM Hospitals of Care and Research, Bari, ${ }^{\mathrm{a}}$ Cotignola, ${ }^{\mathrm{b}}$ Firenze, ${ }^{\mathrm{c}}$ Lecce, ${ }^{\mathrm{d}}$ Palermo, ${ }^{\mathrm{e}}$ Reggio Emilia, ${ }^{\mathrm{f}}$ Rapallo, ${ }^{\mathrm{g}}$ and Torino, ${ }^{\mathrm{h}}$ Italy; The Terlizzi Hospital, ${ }^{\mathrm{i}}$ Terlizzi, Italy; and the University of Palermo, ${ }^{\mathrm{j}}$ Palermo, Italy

Disclosures: Authors have nothing to disclose with regard to commercial support.

Received for publication Feb 24, 2010; revisions received April 7, 2010; accepted for publication May 17, 2010; available ahead of print June 21, 2010.

Address for reprints: Giuseppe Nasso, MD, Department of Cardiac Surgery, GVMHospitals of Care and Research, Anthea Hospital, Via Camillo Rosalba 35-37, 70124 Bari, Italy (E-mail: gnasso@libero.it).

0022-5223/\$36.00

Copyright (c) 2011 by The American Association for Thoracic Surgery doi:10.1016/j.jtcvs.2010.05.010
}

agreement that (1) cardiac operations in the octogenarians are feasible, although at the price of increased operative mortality and morbidity than in younger candidates ${ }^{3,4} ;(2)$ nonelective priority represents a major determinant of poorer outcome $\mathrm{e}^{3-5}$; (3) when coronary disease accompanies valve disease, the prognosis is significantly ameliorated if concomitant coronary artery bypass graft $(\mathrm{CABG})$ surgery is done, as in the younger candidates ${ }^{6}$; and (4) acceptable 5-year survival and quality of life have been reported in limited series. ${ }^{6}$ However, uncertainty remains as to the prognostic gain of combined coronary and mitral valve surgery. Data from the US National Cardiovascular database, which took into analysis 4743 cases, confirmed the concept that in selected octogenarians the operative mortality is similar to that observed in younger surgical candidates. ${ }^{7}$ Such analysis provided only immediate results whereas follow-up data were not available, and patients undergoing surgical procedures on the thoracic aorta had been excluded.

The clinical spectrum of elderly candidates for cardiac surgery is evolving. ${ }^{8}$ Little evidence is available as to the immediate and long-term outcomes of these patients. In addition, the established indicators of surgical risk such as the additive EuroSCORE do not appear to reliably predict 


\author{
Abbreviations and Acronyms \\ AMI = acute myocardial infarction \\ $\mathrm{CABG}=$ coronary artery bypass graft \\ CCS = Canadian Cardiovascular Society \\ $\mathrm{CPB}=$ cardiopulmonary bypass \\ DASI $=$ Duke Activity Status Index \\ GVM = "Gruppo Villa Maria" \\ NYHA $=$ New York Heart Association
}

surgical mortality in elderly patients. ${ }^{9}$ Thus, the currently available data should be updated in the light of the more compound population of elderly individuals now subjected to cardiac surgery. The aim of the present study was 3-fold:

1. Provide an updated picture of baseline features and cardiac surgical indications in a large population of elderly patients

2. Assess the immediate surgical results, the long-term survival, and quality of life

3. Identify the predictors of immediate and long-term outcome

\section{METHODS}

\section{Patient Selection and Data Collection}

Since 1995, the clinical data pertaining to all patients undergoing cardiac surgery within the Gruppo Villa Maria (GVM) Hospitals are prospectively included in an electronic database at the time of discharge. Data pertaining to patients operated on before 1995 (1992-1994 period) were obtained from hospital records and entered retrospectively into the database. As part of a routine quality control program, the database is periodically checked for errors and omissions. Follow-up data are regularly incorporated into the same database: each patient surviving the operation is followed up by outpatient visits and/or telephone interviews 1 month after hospital discharge and every 6 months thereafter. Outpatient visits include physical examination, assessment of New York Heart Association (NYHA) functional class, electrocardiogram, chest radiography, and transthoracic echocardiography. Telephone interviews are conducted according to a standardized format (including survival, functional status, need for hospitalizations, and quality of life).

We queried the database to identify all patients who were 79 years or older at the time of cardiac surgery and were operated on in the period January 1992 to January 2008. No further selection criteria were applied. Patients included in the GVM database were operated on in 8 hospitals located in 8 Italian cities (Turin, Rapallo, Reggio Emilia, Ravenna, Florence, Bari, Lecce, and Palermo).

The institutional review board approved the study protocol. Given the retrospective nature of the investigation, and inasmuch as all patients' data were treated anonymously and no additional diagnostic or therapeutic procedures were conducted, individual informed consent to enter the study was not deemed necessary.

\section{Study End Points}

1. Operative results (mortality/morbidity) in elderly patients undergoing coronary, valvular, thoracic aortic, or combined surgery
2. Five-year follow-up results (mortality/functional recovery/ quality of life) of elderly patients undergoing coronary, valvular, thoracic aortic, or combined surgery

3. Comparison of operative and follow-up results between riskmatched octogenarians and nonagenarians undergoing elective cardiac surgery.

\section{Patient Management}

As part of an institutional policy, a multimodality protocol is used to select the elderly candidates for cardiac surgery. The crude surgical risk, the patient's functional status, social environment and support, and the individual motivation to undergo surgery are evaluated. Surgery is not considered for bedridden patients and for those deprived of a solid family or caregiver support. Calculation of the Duke Activity Status Index (DASI) is part of the evaluation. ${ }^{10,11}$ A DASI score of 10 or less is considered an absolute contraindication to surgery. The predicted surgical risk was calculated using the logistic EuroSCORE model, which has been reported to perform better than the additive model in the higher-risk patient subpopulations. Once surgery is planned, the patient enters an in-hospital preoperative program aimed at optimizing respiratory function and general physical condition. Early and aggressive functional rehabilitation is initiated after surgery, and support is provided by professional therapists. Such programs are pursued following individually tailored schedules. Whenever allowed by the clinical condition, early discharge is encouraged by close interaction with the patients' families and social environments. Nutritional counseling and rehabilitation support are provided after discharge to home. All cardiac departments belonging to the GVM Organization follow this standardized protocol. Details of the program are described elsewhere. ${ }^{12}$

\section{Statistical Methods}

The statistical methods adopted for data collection, analysis, quality control, and interpretation conform to the "Uniform Requirements for Manuscripts submitted to Biomedical Journals" (ICMJE) (International Committee of Medical Journal Editors. Uniform Requirements for Manuscripts submitted to Biomedical Journals. October 2007 updated. www. ICMJE.org).

Continuous and categorical variables are given as mean \pm standard deviation and as percentages, respectively. A multiple logistic regression model was built to identify the predictors of operative mortality. Second, a Cox proportional hazards regression model was created to identify the determinants of late mortality. The assumptions of both models were checked and met, and they were validated with a bootstrap method as well as the Schoenfeld residuals. The following factors were included in the model as potential explanatory variables for operative mortality: NYHA, Canadian Cardiovascular Society (CCS) angina class, age, surgical priority (elective vs nonelective), left ventricular function (ejection fraction: $\geq 50 \%$, good; $<50 \%$ and $>30 \%$, moderately depressed; $\leq 30 \%$, severely depressed), gender, diabetes, hypertension, smoking history, recent acute myocardial infarction (AMI), chronic obstructive pulmonary disease, active endocarditis, crossclamp time, cardiopulmonary bypass (CPB) time, combined mitral and coronary surgery, need for postoperative counterpulsation, and need for postoperative transfusion. The same variables were included in the model as potential predictors of late mortality. Baseline features were defined according to the EuroSCORE criteria (see below). Survival analysis was conducted according to the Kaplan-Meier method, and corresponding curves were built. Curves were compared by the log-rank statistic.

After multivariable testing, the study population was divided into 2 strata on the basis of age at the time of operation: those aged less than 90 years and those aged 90 years or more. Nonelective candidates were excluded from this subanalysis. On the basis of the preoperative risk profile characteristics apart from age, logistic regression was used to model the probability of each patient to enter either the group of patients aged less than 90 or the group of patients aged 90 or more. The variables used for the calculation of the 
TABLE 1. Preoperative demographics and characteristics of study patients $(\mathrm{n}=6,802)$

\begin{tabular}{lrr}
\hline \multicolumn{1}{c}{ Variable } & No. & \multicolumn{1}{c}{$\%$} \\
\hline Sex, male & 3846 & $56 \%$ \\
NYHA functional class III or IV & 2176 & $31.9 \%$ \\
CCS angina class 3 or 4 & 2301 & $33.8 \%$ \\
Recent ( $\leq 90$ d) Q-wave AMI & 1256 & $18.5 \%$ \\
Q-wave AMI within 90 days & 578 & $8.5 \%$ \\
Previous PTCA/stenting & 529 & $7.8 \%$ \\
Diabetes & 1627 & $23.9 \%$ \\
Systemic hypertension & 4947 & $72.7 \%$ \\
Chronic renal insufficiency & 410 & $6 \%$ \\
COPD & 981 & $14.4 \%$ \\
Peripheral arterial disease & 1586 & $23.3 \%$ \\
Active bacterial endocarditis & 18 & $0.3 \%$ \\
LVEF $>30 \%$ and $\leq 49 \%$ & 1700 & $25 \%$ \\
LVEF $<30 \%$ & 226 & $3.3 \%$ \\
Nonelective priority & 1613 & $23.7 \%$ \\
Mean logistic EuroSCORE & $12.1 \pm 2.1$ & \\
\hline
\end{tabular}

NYHA, New York Heart Association functional class; CCS, Canadian Cardiovascular Society class of angina; $A M I$, acute myocardial infarction; $P T C A$, percutaneous transluminal coronary angioplasty; $C O P D$, chronic obstructive pulmonary disease; $L V E F$, left ventricular ejection fraction.

operative risk by means of the EuroSCORE (as available online at www. euroscore.org), except for the patient's age, were included in the model. The propensity score was calculated for all individuals. For each patient aged 90 or more, a matching patient with the closest score was then selected from the larger pool of patients aged less than 90 (maximum allowable difference: 0.1 ). Twenty-four patients aged 90 or more were excluded owing to the lack of matching patients from the other group. Thus, 2 risk-matched subgroups were finally obtained: group A (58 patients, aged $<90$ years at the time of operation) and group B (58 patients, aged $\geq 90$ years). Group comparison was performed by the $\chi^{2}$ test for categorical variables and by the Student $t$ test for continuous variables. Tests were 2-tailed. Yates correction was applied. The alpha level was set at .05 .

Clustering of data by site did not occur (1-way analysis of variance disclosed no statistically significant differences with respect to baseline and operative features among patient subgroups derived from each hospital). The use of standardized clinical and surgical protocols within the hospitals contributing to the present investigation probably accounts for such comparability.

Statistics were performed with SPSS (Statistical Package for Social Sciences) version 11.0 for Windows (SPSS, Inc, Chicago, Ill) and with SAS software for propensity scoring analysis (SAS/STAT version 8; SAS Inc, Cary, NC).

\section{Definitions}

Elective priority: Possibility to discharge the patient from the hospital after the surgical indication has been established, with later readmission and operation according to a planned schedule

Nonelective priority: Need to perform the operation shortly after diagnosis and within the same hospital admission owing to unstable clinical conditions

Recent AMI: AMI occurring within 90 days of the operation

Respiratory insufficiency: $\mathrm{PaO}_{2}$ of $60 \mathrm{~mm} \mathrm{Hg}$ or less on room air and/or need for prolonged ( $>3$ days overall) postoperative mechanical ventilation

Renal failure: Postoperative increase in serum creatinine level of $2 \mathrm{mg} / \mathrm{dL}$ or more compared with the preoperative levels

Infectious complications: Sepsis, deep sternal wound infection, and any proven pulmonary infection
TABLE 2. Operative details and results

\begin{tabular}{lcc}
\hline \multicolumn{1}{c}{ Variable } & No. & $\%$ \\
\hline Pedicled LITA (no. of conduits) & 2290 & $19 \% *$ \\
Pedicled RITA (no. of conduits) & 92 & $0.8 \% *$ \\
Skeletonized LITA (no. of conduits) & 1482 & $12.3 \% *$ \\
Free-graft RITA (no. of conduits) & 607 & $5 \% *$ \\
Radial artery grafts (no. of conduits) & 103 & $0.8 \% *$ \\
Greater saphenous vein grafts (no. of conduits) & 6659 & $55.3 \% \S$ \\
CPB time (min) & $118.4 \pm 32.8$ & - \\
Operative mortality & 232 & $3.4 \%$ \\
ICU LOS (d) & $5.4 \pm 2.0$ & - \\
Respiratory insufficiency & 565 & $8.3 \%$ \\
Acute renal failure & 639 & $9.4 \%$ \\
Neurologic complications & 547 & $8 \%$ \\
Infectious complications & 166 & $2.4 \%$ \\
Gastrointestinal complications & 131 & $1.9 \%$ \\
\hline
\end{tabular}

LITA, Left internal thoracic artery; RITA, right internal thoracic artery; $C P B$, cardiopulmonary bypass; $I C U$, intensive care unit; $L O S$, length of stay. *Percentage of the total number of conduits used.

Gastrointestinal complications: Bowel ischemia, cholecystitis, or gastric ulcer

A neurologic complication was recorded when a new focal deficit or coma, associated with computed tomographic scan demonstration of a recent ischemic lesion, developed either on awakening from anesthesia (intraoperative cerebrovascular accident) or at later postoperative time, after a normal neurologic status had been documented on wakening from anesthesia (postoperative cerebrovascular accident). Stroke or transient ischemic attack was recorded when the neurologic complication lasted, respectively, more or less than 24 hours.

Operative death was defined when occurring within 30 days of the operation or before hospital discharge. Death was considered cardiac in origin if preceded by signs/symptoms of heart disease or failure and if a direct cause-effect link could be reasonably established between the cardiac dysfunction and death.

\section{RESULTS \\ In-Hospital Results}

A total of 6802 patients were selected from the database and included in the analysis. Table 1 outlines the preoperative demographics and characteristics of the study population. Mean age at surgery was $82 \pm 2.1$ years (median: 81 years; range: $79-103$ years). A total of $2176(32 \%)$ patients were in NYHA class III or IV, and 2301 patients (33.8\%) were in CCS class 3 or 4 . Mean additive EuroSCORE was $7.1 \pm 1.3$ (median: 7; range: 4-20), whereas the mean logistic EuroSCORE was $12.1 \pm 2.1$ (median: 12; range: 6-29). Nonelective surgery was performed in $1613(23.7 \%)$ patients; of these, 31 patients underwent a salvage operation.

Table 2 summarizes the operative results. Although the majority of patients were submitted to CABG and/or aortic valve replacement, other operations (mitral, tricuspid, or aortic valve repair and replacement of the thoracic aorta) were performed in a considerable proportion of cases. Details are as follows: valve replacement (aortic, 2365 cases; mitral, 444 cases; tricuspid, 2 cases), valve repair (aortic, 66 cases; mitral, 532 cases; tricuspid, 232 cases), CABG 
TABLE 3. Results of multiple logistic regression: Predictors of operative mortality

\begin{tabular}{lccc}
\hline \multicolumn{1}{c}{ Variable } & $\mathbf{9 5} \% \mathbf{C I}$ & OR & $\boldsymbol{P}$ value \\
\hline Nonelective priority & $1.98-4.7$ & 7.9 & $<.001$ \\
IABPC & $0.78-18.9$ & 2.7 & .004 \\
CPB time & $1.2-9.56$ & 3.0 & .032 \\
Blood transfusion & $0.9-32.7$ & 2.7 & .025 \\
LVEF $<30 \%$ & $1.3-2.8$ & 5.3 & .007 \\
COPD & $0.9-41.8$ & 3.7 & .015 \\
\hline
\end{tabular}

$C I$, Confidence intervals; $O R$, odds ratio. $I A B C P$, intra-aortic balloon counterpusation; $C P B$, cardiopulmonary bypass; $L V E F$, left ventricular ejection fraction; $C O P D$, chronic obstructive pulmonary disease.

(12,034 coronary vessels bypassed), thoracic aortic replacement (ascending, 315 cases; arch, 28 cases). One-vessel coronary revascularization was accomplished in 1026 cases, 1-vessel CABG was done in 1710 cases, and 3-vessel CABG in 1709 cases. The saphenous vein accounted for $55 \%$ of the total coronary grafts implanted. Off-pump coronary surgery was performed in $6.1 \%$ of all patients having CABG.

Overall operative mortality was $3.4 \%$. Death was cardiac in origin in $181(77 \%)$ cases. Operative mortality according to the type of surgery performed was as follows: isolated CABG surgery, $2.5 \%$; coronary and aortic valve surgery (replacement/repair), 3.7\%; coronary and mitral valve surgery (replacement/repair), $4.2 \%$; isolated valvular surgery (aortic and/or mitral and/or tricuspid), 3.3\%; thoracic aortic surgery (with or without any associated procedure), 5.6\%. There were $2760(18.3 \%)$ postoperative complications in 1244 patients. One hundred twenty patients received tracheostomy owing to respiratory failure. Readmission to the intensive care unit was required in 233 cases $(3.4 \%$ of overall population). Regardless of the type of intervention, in the urgent/emergency cases the operative mortality and morbidity were, respectively, $6 \%$ and $49 \%$, both significantly higher than those observed in the elective cases $(P<.001)$.

The following factors were significant predictors of operative mortality at logistic regression: nonelective surgery, need for intra-aortic counterpulsation, CPB time, need for blood transfusion, depressed left ventricular systolic function, and chronic pulmonary disease (Table 3 ).

We conducted a subgroup analysis by comparing the outcomes of patients operated on in the 1992 to 1998 period versus those of patients undergoing surgery in the last half of our database. Operative mortality was slightly lower in the "recent" subgroup (3.3\% vs 3.6\%), although not statistically different $(P=.14)$. Although the rate of infectious complications was increased among the patients operated on during the first period $(P=.03)$, the rates of the remaining postoperative complications were comparable among subgroups.

\section{Follow-up Results}

Follow-up data were available for $6605(97 \%)$ patients, whereas 197 were lost to follow-up (censored observations).

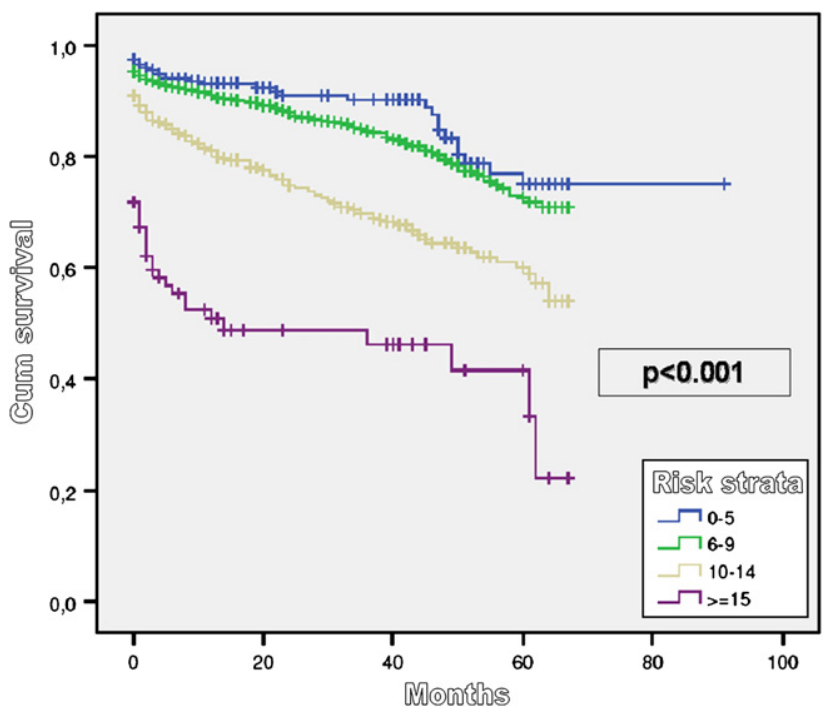

FIGURE 1. Kaplan-Meier survival curves of patients divided according to strata of predicted risk (additive EuroSCORE). $P$ value is from $\log$-rank statistic.

Mean follow-up time was $5.9 \pm 2.7$ years (median, 5.8 years; range, 5 months to 15 years). Cumulative mortality was $7.5 \%$ (495 uncensored observations), and a total of $6110(89.9 \%)$ patients were alive and censored at the end of follow-up.

Mean age of follow-up survivors was $86.1 \pm 2.5$ years (men, $85.0 \pm 1.9$ years; women, $87.0 \pm 1.8$ years). Cox proportional hazards regression analysis identified the following as significant predictors of long-term mortality: moderately/severely depressed preoperative left ventricular function, previous AMI, and combined coronary and mitral surgery (Table E1). Survival curves for patient strata according to the operative risk are reported in Figure 1. Patients with higher predicted mortality who underwent the operation and survived displayed poorer survival during followup $(P<.001)$.

The mean functional capacity significantly improved after surgery in our population and, on average, NYHA functional status decreased by 2 classes (Figure E1). At the end of the study period, a significantly greater number of patients were ub in class I $(P<.0001)$ and a significantly lower number were in classes III $(P<.0001)$ and IV $(P<.0001)$. In fact, most patients were able to sustain mild-to-moderate physical activity and only $5 \%$ were bedridden (Figure E2).

\section{Immediate and Long-Term Results in Nonagenarians}

Propensity-matching of octogenarians and nonagenarians yielded 2 groups (respectively, A and B) of 58 patients each. The clinical results observed in each group are shown in Table 4 . The data suggest that the early outcome of the nonagenarians operated on electively is not significantly different from that of risk-matched octogenarians. Survival is still 
TABLE 4. Operative and follow-up results in group A (octogenarians) versus group B (nonagenarians)

\begin{tabular}{lccc}
\hline \multicolumn{1}{c}{ Variable } & Group A & Group B & $\boldsymbol{P}$ value \\
\hline Operative mortality & $5.2 \%$ & $10.3 \%$ & .48 \\
One-year follow-up mortality & $8.6 \%$ & $20.7 \%$ & .11 \\
Three-year follow-up mortality & $10.3 \%$ & $32.7 \%$ & $<.024$ \\
End of follow-up mortality & $12.1 \%$ & $55.2 \%$ & $<.0001$ \\
NYHA functional class III or IV* & $3.4 \%$ & $5.2 \%$ & .99 \\
\hline NYHA New York Heart Association *NYHA class at last available follow-up visit.
\end{tabular}

comparable at the first postoperative year but rapidly falls from the second postoperative year among the nonagenarians. This is probably due to the very advanced age of group B patients. Actually, noncardiac causes of death were the most frequent in this subpopulation $(68 \%)$.

\section{DISCUSSION}

The increasing age of candidates is a distinctive feature of the current cardiac surgical practice. The elderly are now the fastest growing population in Western countries, and the number of elderly patients $(89.9 \%)$ with disease potentially eligible for surgery is expected to increase. ${ }^{1,2}$ This scenario entails a complex decision-making process that involves ethical, social, and economic issues. Additionally, escalating medical costs nowadays impose a cost-effective medical care. Indeed, this represents a true challenge in elderly patients, who are more likely to have morbid conditions and a prolonged/complicated postoperative course. ${ }^{13}$ Uncertainties also exist regarding the impact of surgery on the long-term prognosis. To the best of our knowledge, the present article takes into analysis the largest series published so far, except for national databases, of elderly and very elderly patients subjected to cardiac operations. The present findings may contribute to dedicated guidelines for the practice of cardiac surgery in elderly patients.

\section{Early Mortality and Morbidity}

We observed a mean operative mortality $(3.4 \%)$ considerably lower than reported by previous investigators. ${ }^{14} \mathrm{In}$ other experiences, the bulk of hospital deaths occurred after urgent/emergency operations $(87.5 \%)$, although mortality after elective surgery was still considerable $(7.5 \%))^{9}$ It is reported that octogenarians have a 3-fold increased risk of mortality compared with younger patients. ${ }^{15,16}$

Multiple reasons probably account for the better immediate outcome observed in our patients, including the recent improvements of surgical techniques, myocardial protection, and postoperative care. The multimodality protocol based on careful patient characterization and selection has probably contributed to this result. Thus, the logistic preoperative EuroSCORE (mean 12.1 in our series) is not adequate to reliably predict the risk in this population and should not be considered a determinant element in surgical decision-making. EuroSCORE has been reported to overestimate in the higher-risk categories. ${ }^{9}$ In this context, the array of predictors of mortality could involve socioeconomic, psychologic, and biological parameters. ${ }^{17,18}$ Hence, the development of dedicated risk scores may be advisable. Our results basically are in agreement with these concepts, inasmuch as the predictors of operative mortality were predominantly patient-related factors. The duration of CPB time and the need for blood transfusion may partially reflect the complexity of the intervention. A meticulous and expeditious surgical technique, optimal myocardial protection and careful tissue handling, minimization of CPB time, and blood loss appear important in dictating outcome. In our experience, a nonelective indication was associated with a nearly 8 -fold increased risk of operative mortality, in agreement with previous evidence. ${ }^{6}$ This may argue against the conservative approach that is often used in elderly subjects. Conversely, postponing a potentially successful elective intervention may result in the necessity of facing the complications and the poor outcome of emergency surgery. We note a wide use of arterial conduits in our population. This may result from the limited availability of the saphenous vein as a conduit in elderly patients (poor leg vein quality or previous saphenectomy) but also reflects our policy to encourage a liberal use of arterial conduits (mainly internal thoracic arteries) even in the elderly patients.

Inasmuch as the nonelective priority is the main determinant of operative mortality, we decided to exclude the nonelective cases from the propensity-matched comparison between octogenarians and nonagenarians. We believe that the analysis of the sole elective cases yields the most useful indications to the clinician, who is faced with the difficult decision-making in elderly candidates. Indeed, our intergroups comparison revealed that the early outcome of nonagenarians undergoing cardiac surgery is not significantly different from that observed in risk-matched octogenarians.

\section{Late Survival and Functional Status}

Our data confirm previous findings that cardiac operations in elderly patients may provide favorable long-term survival and functional recovery. At the end of follow-up, nearly $90 \%$ of the patients were alive, and NYHA status was improved by a median of 2 classes. The majority of patients were able to sustain mild-to-moderate physical activity, and only a limited proportion were bedridden and completely dependent on caregivers. In a pooled analysis, the latter patients were more likely to have depressed ejection fraction and chronic obstructive lung disease at the time of surgery. The same individuals frequently required prolonged intensive care unit stay and tracheostomy postoperatively.

The lack of a matched, medically treated control group represents a limitation to our conclusions over the prognostic 
impact of cardiac surgery. Nevertheless, in our series at the end of follow-up the mean age of the survivors was considerably greater than predicted by demographic national registries. The Cox analysis underlines that patient-related factors, such as myocardial function and the general clinical conditions, are the main midterm and long-term outcome determinants of elderly patients. In this respect, elderly patients are not different from the general surgical population. We emphasize that the evaluation of functional indexes is fundamental for the selection of surgical candidates: in our experience the DASI score was reliably used for this purpose. Although correlated even with operative mortality, this health-related quality of life scoring system has been reported to reliably predict a prolonged hospital stay, postoperative morbidity, ${ }^{19,20}$ and the long-term survival of elderly candidates. ${ }^{21}$ This is particularly evident in the elderly candidates, thus underlining the importance of the functional status in dictating the surgical outcome in this population. The DASI score for each patient was integrated with calculation of the EuroSCORE and overall clinical/environmental evaluation in the decision-making of individual candidates. Finally, the patients undergoing combined coronary and mitral surgery in our series had a less favorable late prognosis than those receiving isolated coronary or valve surgery. The subtending mechanisms are widely recognized in the general surgical population and include a more advanced disease, poorer ventricular function, and the difficulties in repairing ischemic mitral incompetence. This finding should be carefully considered while evaluating elderly candidates for surgery.

As an observational finding, we note that the mean age of follow-up survivors in our series is greater than the life expectancy for Italy according to (1) European databases (life expectancy for men: 80.37 years; life expectancy for women: 85.31 years), year $2005,{ }^{22}$ and (2) Italian national databases (life expectancy for men: 80.1 years; life expectancy for women: 85.7 years) [ISTAT, Istituto Nazionale di Statistica, www.istat.it].

\section{CONCLUSIONS}

The major drawback of our study is represented by its retrospective, observational nature. However, the reliability of our conclusions is strengthened by the use of similar protocols for both patient selection and operative/postoperative care within all the contributing centers. The observation of acceptable operative mortality and improvement in functional capacity and quality of life during the follow-up encourages a relatively liberal use of cardiac surgery in elderly and very elderly patients. However, accurate selection of candidates, functional evaluation, and a multidisciplinary customized management are mandatory. ${ }^{12}$ The encouraging results depicted herein should therefore be interpreted as the result of careful patient selection and meticulous preoperative, intraoperative, and postoperative multimodality management. Thus, our conclusions can be difficultly generalized to the broad population of elderly individuals with severe heart disease. Such shortcomings may be addressed by a comparison between the present population of elderly patients and a risk-matched cohort of surgical candidates. As such, a direct comparison between our population and an age- and gender-matched cohort of the Italian population would add greatly interesting information. We acknowledge the lack of both these comparisons as a limitation to our conclusions, and we advise the availability of national data sets to conduct such investigations.

Elderly and very elderly subjects should not be denied cardiac surgery on the basis of advanced age alone. Cardiac operations can be routinely performed with acceptable mortality and favorable long-term results in selected patients. The EuroSCORE appears to overestimate the operative risk and should be carefully interpreted while the individual patients are being evaluated for surgery. Earlier operations should be encouraged, to avoid nonelective presentations. As well, the relatively poor long-term outcome of combined coronary and mitral surgery should be carefully considered. Specific guidelines for cardiac surgery in elderly patients may be advisable.

The finding of optimal results of cardiac surgery in these elderly candidates, who may be considered to have excessive operative risk, should prompt a reflection over the potential for the application of the percutaneous valve technologies currently under development. A word of caution must be expressed over the expansion of indications for the transcatheter methods to the octogenarians and nonagenarians with medium/low predicted risk.

\section{References}

1. Greengross S, Murphy E, Quam L, Rochon P, Smith R. Aging: a subject that must be at the top of the world agendas. BMJ. 1997;315:1029-30.

2. US Bureau of the Census. Statistical abstract of the United States. ed. 110. Washington DC: US Government Printing Office; 1990.

3. Gehlot A, Mullany CJ, Ilstrup D, Schaff H, Orszulak T, Morris J, et al. Aortic valve replacement in patients aged eighty years and older: early and long-term results. J Thorac Cardiovasc Surg. 1996;111:1026-36.

4. Kohl P, Kerzmann A, Lahaye L, Gerard P, Limet R. Cardiac surgery in octogenarians-perioperative outcome and long-term results. Eur Heart J. 2001;22:1235-43.

5. Kohl P, Kerzmann A, Honore C, Comte L, Limet R. Aortic valve surgery in octogenarians: predictive factors for operative and mid-term results. Eur J Cardiothorac Surg. 2007;31:600-6.

6. Melby SJ, Zierer A, Kaiser SP, Guthrie T, Keune J, Schuessler R, et al. Aortic valve replacement in octogenarians: risk factors for early and late mortality. Ann Thorac Surg. 2007;83:1651-7.

7. Alexander KP, Anstrom KJ, Muhlbaier LH, Grosswald R, Smith P, Jones R, et al. Outcomes of cardiac surgery in patients $>$ or $=80$ years: results from the National Cardiovascular Network. J Am Coll Cardiol. 2000;35:731-8.

8. Ailawadi G, Swenson BR, Girotti ME, Gazoni L, Peeler B, Kern J, et al. Is mitral valve repair superior to replacement in elderly patients? Ann Thorac Surg. 2008; 86:77-85.

9. Zingone B, Pappalardo A, Dreas L. Logistic versus additive EuroSCORE. Comparative assessment of the two models in an independent population sample. Eur J Cardiothorac Surg. 2004;26:1134-40.

10. Hlatky MA, Boineau R, Higginbotham MB, Lee KL, Mark DB, Califf RM, et al. A brief self-administered questionnaire to determine functional capacity (the Duke Activity Status Index). Am J Cardiol. 1989;64:651-4. 
11. Koch GC, Li L, Shishehbor M, Nissen S, Sabik J, Starr N, et al. Socioeconomic status and comorbidity as predictors of preoperative quality of life in cardiac surgery. J Thorac Cardiovasc Surg. 2008;136:665-72.

12. Speziale G, Bonifazi R, Cavagnaro $\mathrm{P}$, Di Gregorio $\mathrm{O}$, Pasquè $\mathrm{A}$, Zanardi $\mathrm{S}$, et al. Vantaggi di un approccio multidimensionale personalizzato al paziente anziano sottoposto a chirurgia cardiaca: risultati a breve e lungo termine su 193 pazienti. Ital Heart J Suppl. 2005;6:674-81.

13. Likosky DS, Dacey LJ, Baribeau YR, Leavitt B, Clough R, Cochran R, et al., Northern New England Cardiovascular Disease Study Group. Long-term survival of the very elderly undergoing coronary artery bypass grafting. Ann Thorac Surg. 2008;85:1233-8.

14. Avery GJ, Ley SJ, Hill JD, Hershon JJ, Dick SE. Cardiac surgery in the octogenarian: evaluation of risk, cost and outcome. Ann Thorac Surg. 2001;71:591-6.

15. Craver JM, Puskas JD, Weintraub WW, Shen Y, Guyton RA, Gott JP, et al. 601 octogenarians undergoing cardiac surgery: outcome and comparison with younger age group. Ann Thorac Surg. 1999;67:1104-10.

16. Peterson ED, Cowper PA, Jollis JG, Bebchuk JD, DeLong ER, Muhlbaier LH, et al. Outcomes of coronary artery bypass surgery in 24,461 patients aged 80 years or older. Circulation. 1995;92(Suppl 2):85-91.
17. Oxman TE, Freeman DH, Manheimer ED. Lack of social participation or religious strength and comfort as risk factors for death after cardiac surgery in the elderly. Psychosom Med. 1995;57:5-15.

18. Rumsfeld JS, Magid DJ, O’Brien M, McCarthy M Jr, MaWhinney S, Shroyer AL et al. Changes in health-related quality of life in following coronary artery bypass graft surgery. Ann Thorac Surg. 2001;72:2026-32.

19. Ho PM, Masoudi FA, Peterson PN, Shroyer AL, McCarthy M Jr, Grover FL, et al. Health-related quality of life predicts mortality in older but notin younger patients following cardiac surgery. Am J Geriatr Cardiol. 2005;14:176-82.

20. Curtis LH, Phelps CE, McDermott MP, Rubin HR. The value of patient-reported health status in predicting short-term outcomes after coronary artery bypass graft surgery. Med Care. 2002;40:1090-100.

21. Koch CG, Li L, Lauer M, Sabik J, Starr NJ, Blackstone EH. Effect of functional health-related quality of life on long-term survival after cardiac surgery. Circulation. 2007;115:692-9.

22. Jagger C, Gillies C, Moscone F, Cambois E, Van Oyen H, Nusselder W, et al. Inequalities in healthy life years in the 25 countries of the European Union in 2005: a cross-national meta-regression analysis. Lancet. 2008;372: 2124-31. 


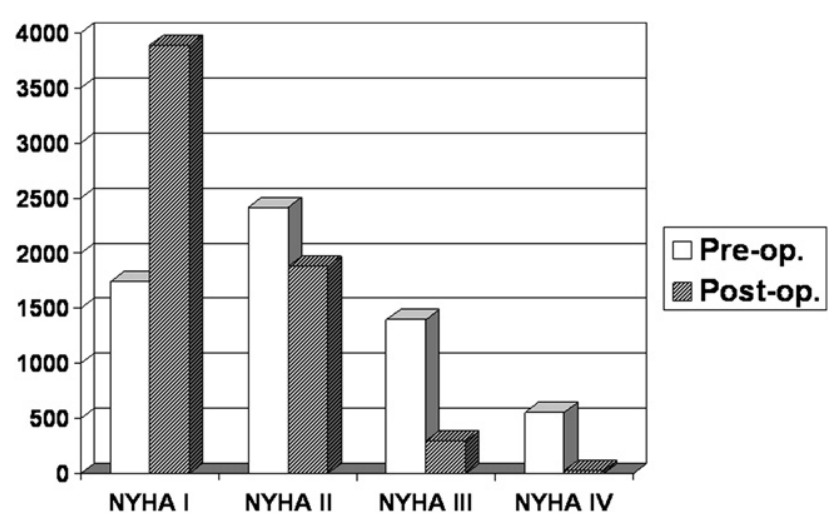

FIGURE E1. Bar diagram showing New York Heart Association (NYHA) functional classification among the patients who were alive at the end of follow-up.

TABLE E1. Results of Cox proportional hazards regression model: predictors of follow-up mortality

\begin{tabular}{lllc}
\hline \multicolumn{1}{c}{ Variable } & \multicolumn{1}{c}{$\mathbf{9 5} \%$ CI } & $\boldsymbol{P}$ value & Wald $\boldsymbol{\chi}^{2}$ \\
\hline Moderately/severely depressed LVEF & $1.10-1.46$ & .0002 & 28.36 \\
Previous myocardial infarction & $1.28-2.4$ & .0032 & 25.1 \\
Combined coronary and mitral surgery & $2.36-6.16$ & .009 & 5.5 \\
\hline$C I$, Confidence intervals. $L V E F$, left ventricular ejection fraction.
\end{tabular}

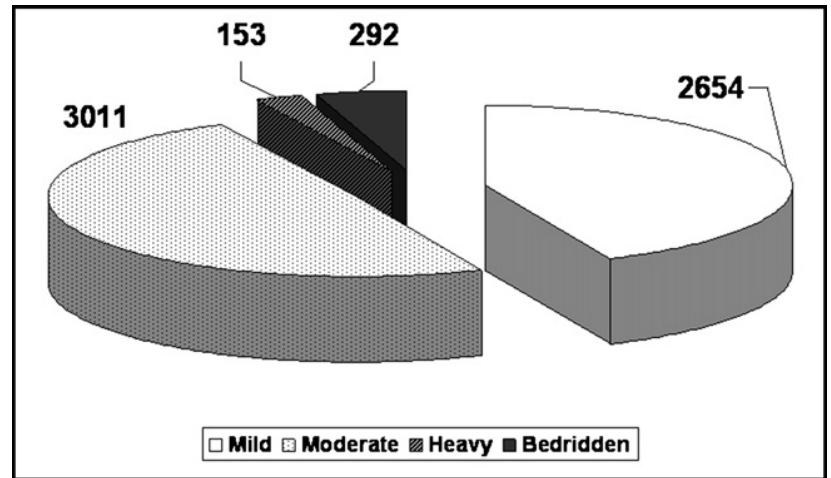

FIGURE E2. Degree of physical activity among patients who were alive at the end of follow-up.

731.e1 The Journal of Thoracic and Cardiovascular Surgery • March 2011 